\title{
A transient three-plasmid expression system for the production of high titer retroviral vectors
}

\author{
Yuko Soneoka, Paula M. Cannon, Emma E. Ramsdale, Joanne C. Griffiths, Gaetano \\ Romano, Susan M. Kingsman and Alan J. Kingsman*
}

\author{
Retrovirus Molecular Biology Group, Department of Biochemistry, University of Oxford, South Parks Road, Oxford \\ OX1 3QU, UK
}

Received October 19, 1994; Revised and Accepted January 11, 1995

\begin{abstract}
We have constructed a series of MLV-based retroviral vectors and packaging components expressed from the CMV promoter and carried on plasmids containing SV40 origins of replication. These two features greatly enhanced retroviral gene expression when introduced into cell lines carrying the SV40 large T antigen. The two packaging components, gag-pol and env, were placed on separate plasmids to reduce helper virus formation. Using a highly transfectable human cell line and sodium butyrate to further increase expression of each component, we achieved helper-free viral stocks of $\sim 10^{7}$ infectious units/ml by $48 \mathrm{~h}$ after transient co-transfection with the three plasmid components. This system can be used both for the generation of high titer retroviral stocks for transduction and for the rapid screening of a large number of MLV gag-pol or env mutants.
\end{abstract}

\section{INTRODUCTION}

The generation of high titer retroviral stocks for the efficient transduction of target cells is an important technical goal for a range of gene transfer applications. Most standard methods currently use cell lines expressing the gag-pol and env genes of MLV (the packaging components). These will package a retroviral vector genome introduced by transduction or by transfection with an appropriate plasmid (1). In order to obtain retroviral stocks of the highest titers, it is necessary to establish additional cell lines that not only contain the gag-pol and env cassettes, but also have the proviral vector genome stably integrated. To identify the highest producing lines, many subclones may then need to be screened, as greatly varying titers are observed between different subclones $(2,3)$. This screening process can take several weeks and the cell lines so established may lose their packaging ability as they are passaged (4). A simple system for the production of retroviral stocks without the use of packaging lines has been described by Landau and Littman (5). This approach exploits the high level of transient expression when COS-7 cells are transfected by plasmids containing SV40 origins of replication by placing both the packaging components and the retroviral vector genome on plasmids carrying such sequences. Following simultaneous transfection of gag-pol, env and vector genome plasmids into COS-7 cells, viral titers of $10^{4}$ infectious units/ml were achieved 48-72 h post-transfection. Retrovirus production by this three-plasmid co-transfection system is a particularly convenient method for the rapid analysis of MLV gag-pol or env mutants and has the advantage of being simple and reproducible in the hands of different operators. However, compared with selected lines, the titers are low.

We report here a simple system for the production of high titer helper-free retroviral vectors in which the powerful CMV promoter $(6,7)$ drives expression of both the packaging components and the genome. These plasmids also contain the SV40 origin of replication. The simultaneous introduction of these plasmids into a cell line expressing the SV40 large T antigen followed by sodium butyrate treatment produced helper-free retrovirus stocks with titers of $\sim 10^{7}$ infectious units $/ \mathrm{ml}$ at $48 \mathrm{~h}$ post-transfection, which were significantly higher than those obtained using vectors driven by the viral $5^{\prime}$ LTR and are as high as dedicated/selected cell lines expressing all three components.

\section{MATERIALS AND METHODS}

Cell lines

NIH 3T3 and 293T (293ts/A1609) (8) cells were maintained in Dulbecco's modified Eagle's medium (GIBCO) containing $10 \%$ (v/v) fetal calf serum and supplemented with antibiotics. 293T cells were obtained from D. Baltimore (Rockefeller University).

\section{Construction of expression plasmids}

The standard gag-pol and $e n v$ plasmids from which we derived our molecules were pgag-polgpt (9) and penv (9). Both plasmids express the viral components from the MLV 5' LTR and pgag-polgpt additionally contains an SV40 origin of replication (ori) and a gpt selectable marker. The retroviral genome vectors used were pLNSX (10), which carries neomycin resistance (neo) and pLZSN (11), which carries neo and lacZ genes. All of these plasmids were obtained from A. D. Miller (Fred Hutchinson Cancer Research Center).

These original plasmids were modified to produce a set of plasmids containing the SV40 ori. A 350 bp BamHI-HindIII

\footnotetext{
${ }^{*}$ To whom correspondence should be addressed
} 
fragment carrying the SV40 ori was inserted into the BamHIHindIII sites in the backbone of the retroviral vector pXTI (12) (Stratagene). This intermediate molecule, designated pRV51, was created to facilitate the introduction of the SV40 ori into any retroviral vector. A backbone EcoRI-HindIII fragment from pRV51 containing the SV40 ori was used to replace the EcoRI-HindIII penv backbone sequences to create plasmid pRV106 (Fig. 1A). The backbone sequences of pRV51 were also used to construct a universal plasmid which functions as a cassette to insert the SV40 ori and to replace the viral promoter within the $5^{\prime}$ LTR with the CMV promoter (see below) in a number of retroviral vectors, such as pLNCX (10), which contain neither element. This universal plasmid was also used to construct the CMV promoter-driven vectors for pLNSX and pLZSN (see below), although both already contain the SV40 ori sequences. Therefore, the backbone sequences of pLNSX and pLZSN were also replaced with the backbone of pRV51, thereby encoding two SV40 ori sequences in the same plasmid. The ScaI site in the backbone ampicillin gene and the KpnI site in the 3' LTR of pRV51, pLNSX and pLZSN were used to insert the backbone sequences of pRV51 into the vector genomes, producing plasmids pRV113 and pRV114 (Fig. 1A).

Expression plasmid pHIT60 is a CMV-driven gag-pol expression plasmid and pHIT50 and pHIT 123 are CMV-driven ecotropic env expression plasmids (Figs 1A and 3A). All three plasmids contain the SV40 ori. Plasmid pGWIHG is a CMV-promoter expression plasmid containing intron A (13) and the SV40 ori. A $2.3 \mathrm{~kb}$ EcoRI-NarI fragment containing the CMV promoter plus intron A unit was excised from pGWIHG, the NarI end was converted to an EcoRI end with a linker and the resulting fragment was used to replace the MLV promoter in pgag-polgpt to produce $\mathrm{pHIT} 60$. For $\mathrm{pHIT} 50$, the $2.4 \mathrm{~kb} E c o \mathrm{RI}$ fragment from penv containing the ecotropic envelope gene was inserted directly into the $E c o$ RI site of pGWIHG. pHIT123 was created to remove the 3' LTR sequences found in pHIT50. A PCR reaction was performed on penv to isolate the p15e env sequences, creating a $B a m \mathrm{HI}$ and $E c o \mathrm{RI}$ site immediately after the env stop codon. The 570 bp NcoI-EcoRI fragment (coordinates 7228-7723) (14) from the PCR product was used to replace the NcoI-EcoRI fragment containing the $3^{\prime}$ end of the env and the $3^{\prime}$ LTR sequences in pHIT50.

The retroviral vectors were manipulated to replace the entire $5^{\prime}$ $\mathrm{U} 3$ region with the $\mathrm{CMV}$ promoter while preserving the authentic RNA start site at the beginning of the MSV R region. A double-stranded oligonucleotide was constructed to span the junction from the SacI site at -13 in the CMV promoter to the KpnI site at +36 in the MSV R sequence (Fig. 1B) and inserted into the cloning vector pSP46 (15). The remaining CMV promoter sequences were restored by inserting the $531 \mathrm{bp}$ $X b a \mathrm{I}-S a c I$ fragment from pKV461 (16) into pSP46, upstream of this synthetic oligonucleotide. This heterologous $5^{\prime}$ unit was excised from pSP46 using the PvuI site in the backbone ampicillin gene and the $K p n I$ site at the end of the oligonucleotide fragment. This was joined to the $P v u I-K p n I$ backbone fragment of pRV51 using the $P v u \mathrm{I}$ site in the ampicillin gene and the $K p n I$ site in the 3' retroviral LTR to create plasmid pRV109. This plasmid functions as a general cassette for the introduction of retroviral vector sequences utilizing the $K p n I$ sites in the $5^{\prime}$ and $3^{\prime}$ LTRs. The vector sequences from pLNSX and pLZSN were inserted into pRV109 in this manner. A CMV promoter-driven vector in which the luc gene was inserted into pLNCX has also

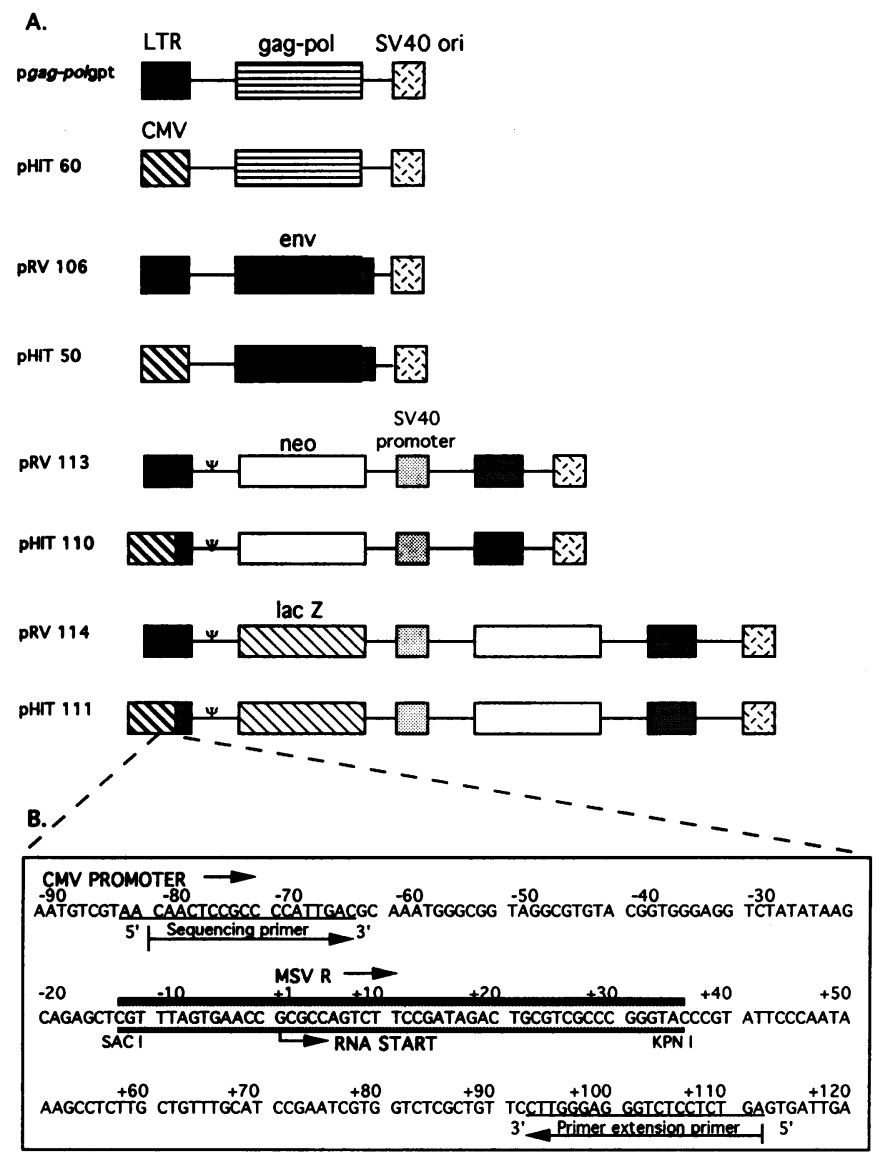

Figure 1. Schematic representation of retroviral constructs used in this study. (A) The retroviral plasmids used in this study are shown schematically. The gag-pol, env and vector components are all shown as LTR-driven constructs (pgag-polgpt, pRV106, pRV113 and pRV114) and their CMV promoter-driven counterparts (pHIT60, pHIT50, pHIT110 and pHIT111). All plasmids contain SV40 origins of replication. pRV106 and pHIT50 contain the ecotropic envelope sequences from plasmid penv. pRV113 and pHIT110 are derived from the retroviral vector pLNSX (10), and pRV114 and pHIT 111 are derived from pLZSN (11). (B) The DNA sequence around the transcriptional start site for pHIT110 and pHIT111 is shown. The oligonucleotide constructed to span the sequence from the SacI site in the CMV promoter to the KpnI site in the retroviral $\mathrm{R}$ region is boxed. Also shown are the positions of the primers used in the primer extension analysis of the transcriptional start site (Fig. 2).

been similarly constructed (data not shown). The CMV promoterdriven vector derived from $\mathrm{pLNSX}$ was designated $\mathrm{pHIT} 110$ and the vector derived from pLZSN was designated pHIT111 (Fig. 1A).

\section{Transient transfections, infections and determination of viral titer}

Transient transfections were performed on 293T cells by overnight calcium phosphate treatment (17) on $10 \mathrm{~cm}$ dishes seeded the previous day to give a maximum of $70 \%$ confluence/ plate on the day of transfection. Ten micrograms of each plasmid (30 $\mu \mathrm{g}$ total) was used for each transfection. Transfection efficiencies of the 293T cells were routinely $70-90 \%$, as determined using a lacZ-containing vector plasmid. The transfected cells were incubated for 15-17 h, after which the medium was replaced with $5 \mathrm{ml}$ of fresh medium. Unless otherwise indicated, the viral supernatants were harvested $48 \mathrm{~h}$ after 
transfection, filtered through $0.45 \mu \mathrm{m}$ filters and titered by infection on NIH 3T3 cells.

NIH 3T3 cells were plated onto six-well culture dishes at a density of 1-2 $\times 10^{5}$ cells/well the day before infection. Supernatant ( $1 \mathrm{ml}$ at appropriate dilutions of the original stock) was added to each well with $8 \mu \mathrm{g} / \mathrm{ml}$ Polybrene (Sigma) and incubated for $2 \mathrm{~h}$, followed by the addition of $1 \mathrm{ml}$ of fresh medium. Twenty four hours later the cells were split 1:4 and seeded onto $5 \mathrm{~cm}$ dishes. Viral titer was determined by selection in $400 \mu \mathrm{g} / \mathrm{ml} \mathrm{G} 418$ (GIBCO) or by X-gal staining. For G418 selection, the medium was replaced every 3 or 4 days and the resistant colonies were counted 10-15 days post-infection by fixing and staining with $0.3 \%$ crystal violet in $70 \%$ methanol. $\mathrm{X}$-gal staining was performed $24 \mathrm{~h}$ following the 1:4 split as previously described (18). Virus titer was determined by multiplying the total number of G418-resistant colonies (or $\beta$-galactosidase-positive cells) by two, to account for the 2 -fold increase in cells during the infection period and the 1:4 split.

\section{Sodium butyrate $(\mathrm{NaB})$ inductions}

$\mathrm{NaB}$ was prepared as a $500 \mathrm{mM}$ stock solution, $\mathrm{pH} 7.0$, sterilized by filtration and added directly to the medium after removal of the calcium phosphate-DNA co-precipitate at final concentrations of $2,5,7$ and $10 \mathrm{mM}$. The cells were treated for $12-14 \mathrm{~h}$, washed with PBS to remove any contaminating $\mathrm{NaB}$ and refed with $5 \mathrm{ml}$ of fresh medium. Viral supernatants were harvested $12 \mathrm{~h}$ later (48 $h$ post-transfection) and subsequently harvested at 60,72 and 84 $h$ after transfection. Viral supernatants were titered on NIH 3T3 cells as described above. Control plates were transfected without $\mathrm{NaB}$ and harvested $48 \mathrm{~h}$ post-transfection.

\section{Helper virus assays}

Transfections were carried out on 293T cells as described above and undiluted viral supernatants were used to infect NIH 3T3 cells. All infections were performed in the presence of $8 \mu \mathrm{g} / \mathrm{ml}$ Polybrene (Sigma). The NIH 3T3 cells were passaged every 4-5 days, when the culture supernatants were filtered and used to infect fresh NIH 3T3 cells. These cells were selected in G418 for up to 10-15 days to assay for the presence of replication-competent virus. In addition, a marker rescue assay was performed using NIH 3T3 cells harboring an integrated lac Z vector derived from pHIT111. These cells were infected with $1 \mathrm{ml}$ of the culture supernatants from the infected NIH 3T3 cells. Twenty four hours later the supernatants were removed and the cells were incubated with $1 \mathrm{ml}$ of fresh medium for another $24 \mathrm{~h}$. Supernatants from these cultures were then used to infect fresh NIH 3T3 cells which were stained for $\beta$-galactosidase activity $24 \mathrm{~h}$ later.

\section{Primer extension analysis}

Total RNA was extracted from 293T cells and analyzed by primer extension, essentially as previously described (19). An oligonucleotide complementary to the $5^{\prime}$ U5 region of the retroviral vector and located 112 bases from the predicted RNA start site (Fig. 1B) was end-labeled with $[\gamma-32 \mathrm{P}] \mathrm{ATP}(5000 \mathrm{Ci} / \mathrm{mmol}$; Amersham) and hybridized to $10 \mu \mathrm{g}$ total RNA at $37^{\circ} \mathrm{C}$ in $40 \mathrm{mM}$ PIPES, pH 6.5, 0.4 M NaCl, 1 mM EDTA for $16 \mathrm{~h}$. Primer extension was carried out at $42^{\circ} \mathrm{C}$ for $1 \mathrm{~h}$ with $10-15 \mathrm{U} / \mathrm{sample}$ AMV reverse transcriptase (Pharmacia). The primer extension products were analyzed on a $6 \%$ denaturing polyacrylamide gel,
Table 1. Comparison of virus titers after transfection of $293 \mathrm{~T}$ cells with LTR-driven and CMV-driven plasmids

\begin{tabular}{|c|c|c|c|}
\hline \multirow[b]{2}{*}{ PROMOTER } & \multirow[b]{2}{*}{ PLASMIDS } & \multicolumn{2}{|c|}{ TITER $^{\mathbf{S}}$} \\
\hline & & $\mathrm{CFU} / \mathrm{ML}^{a}$ & $\mathrm{LFU} / \mathrm{ML}^{b}$ \\
\hline \multirow{2}{*}{$\begin{array}{l}\text { LTR } \\
\text { (Original } \\
\text { plasmids) }\end{array}$} & pgeg-polgpt, penv ${ }^{*}$, pLNSX & $1.8 \times 10^{4}$ & $\mathrm{~N} / \mathrm{A}^{c}$ \\
\hline & pgeg-polgpt, penv, pLZSN & $9.8 \times 10^{3}$ & $1.1 \times 10^{4}$ \\
\hline \multirow{2}{*}{$\begin{array}{l}\text { LTR } \\
(+ \text { SV40 on })\end{array}$} & pgag-polgpt, pRV 106, pRV 113 & $6.0 \times 10^{5}$ & N/A \\
\hline & Pgag-polgpt, pRV 106, pRV 114 & $1.3 \times 10^{5}$ & $7.0 \times 10^{4}$ \\
\hline \multirow{3}{*}{$\begin{array}{l}1 \text { CMV/ } \\
2 \text { LTR }\end{array}$} & pHIT 60, pRV 106, pRV 114 & $\mathrm{ND}^{d}$ & $3.0 \times 10^{4}$ \\
\hline & pgag-polgpt, pHIT 50, pRV 114 & ND & $1.5 \times 10^{5}$ \\
\hline & pgag-polgpt, pRV 106, pHIT 111 & ND & $6.0 \times 10^{4}$ \\
\hline \multirow{3}{*}{$\begin{array}{l}1 \text { LTR / } \\
2 \text { CMV }\end{array}$} & pHIT 60, pHIT 50, pRV 114 & ND & $3.0 \times 10^{5}$ \\
\hline & pgag-polgpt, pHIT 50, pHIT 111 & ND & $2.0 \times 10^{5}$ \\
\hline & pHIT 60, pRV 106, pHIT111 & ND & $4.0 \times 10^{4}$ \\
\hline \multirow[t]{2}{*}{ CMV } & pHIT 60, pHIT 50, pHIT110 & $4.6 \times 10^{6}$ & N/A \\
\hline & pHIT 60, pHIT 50, pHIT 111 & $1.7 \times 10^{6}$ & $1.4 \times 10^{6}$ \\
\hline
\end{tabular}

$\S$ Titers were averages obtained from three independent experiments. There was no more than $15 \%$ variation between experiments.

* Constructs not containing SV40 ori.

${ }^{a}$ Colony forming units per milliliter (G418 resistant colonies).

${ }^{b}$ LacZ forming units per milliliter ( $\beta$-gal positive cells).

$c$ Not applicable.

$d$ Not done.

alongside a sequencing reaction performed on pRV109 with a primer hybridizing within the CMV promoter between -82 and -63 (Fig. 1B).

\section{RESULTS}

\section{Three-plasmid co-transfections to generate retrovirus stocks}

In the first instance, we transfected a standard set of gag-pol, env and vector genome plasmids into 293T cells and titered the resulting viruses on NIH 3T3 cells. The original packaging components used were pgag-polgpt (9), a gag-pol expression vector and penv (9), an ecotropic envelope expression vector. In both plasmids, expression of the viral components is driven by the MLV LTR promoter. The retroviral vector genomes used were pLNSX (10), which carries a neomycin resistance gene, and pLZSN (11), which carries neomycin resistance and a lacZ gene. When these original plasmids were co-transfected into the highly transfectable 293T cells, we produced retrovirus stocks with titers of $1.4 \times 10^{4}$ c.f.u. $/ \mathrm{ml}$ for vector $\mathrm{pLNSX}$ and $9.8 \times 10^{3} \mathrm{l}$.f.u. $/ \mathrm{ml}$ (as assayed by staining for $\beta$-galactosidase) to $1.1 \times 10^{4}$ c.f.u. $/ \mathrm{ml}$ (G418 selection) for vector pLZSN (Table 1). The slightly lower titers obtained by the vector genome pLZSN have been noted previously (11).

\section{Addition of SV40 ori sequences and the CMV promoter boosts viral titers}

293T cells express the SV40 large T antigen (8), which increases the copy number of plasmids containing the SV40 ori. This 


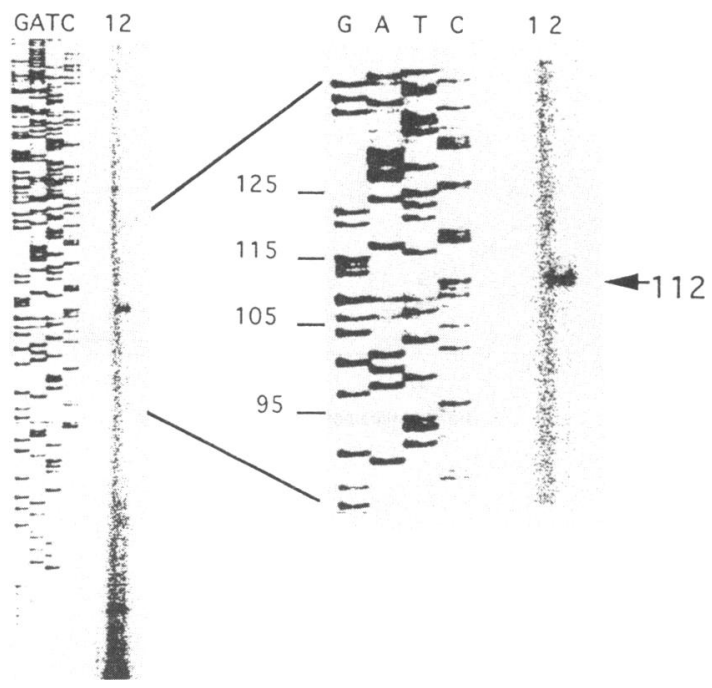

Figure 2. Primer extension analysis of the transcriptional start site in the hybrid CMV-MLV retroviral vectors. Total RNA was extracted from 293T cells and analyzed by primer extension. The primer extension products from untransfected 293T cells (lane 1) and 293T cells transfected with plasmid pHIT110 (lane 2 ) were analyzed on a $6 \%$ denaturing polyacrylamide gel, alongside a sequencing reaction performed on $\mathrm{pRV} 109$ with a primer hybridizing within the CMV promoter between -82 and -63 (Fig. 1B). The primer extended to yield a 112 base product (arrowed) which corresponds to the distance to the predicted start site of the retroviral transcript.

template amplification has been previously used to increase retrovirus titers by transient transfection of COS-7 cells (5). We therefore decided to reproduce this observation with the specific system used here. An SV40 ori was inserted into penv, pLNSX and pLZSN to produce plasmids pRV106, pRV113 and pRV114 respectively (Fig. 1A). Co-transfection of pgag-polgpt, pRV106 and pRV113 or pRV114 into 293T cells increased the infectious titer by at least 14-fold compared to the original set of plasmids, producing more than $10^{5}$ infectious units/ml (Table 1). As before, the titers obtained from the neo-lacZ vector pRV114 were slightly lower than those obtained with the neo vector pRV113. The placement of two SV40 ori sequences in pRV113 and pRV114 (see Materials and Methods) did not significantly alter the virus titers, as comparable titers were obtained when pgag-polgpt, pRV106 and pRV114 or pLZSN were co-transfected (data not shown).

In a further attempt to boost the titers, we decided to improve expression of the packaging components by placing the gag-pol and $e n v$ genes under the control of the powerful CMV promoter. Expression plasmids pHIT60 and pHIT50 are similar to pgagpolgpt and pRV106 respectively, except that the viral genes are under the control of the CMV promoter (Fig. 1A). Transfection of pHIT60, pHIT50 and pRV114 into 293T cells produced virus stocks with a higher titer of $3 \times 10^{5}$ l.f.u. $/ \mathrm{ml}$, compared to the titer of $7 \times 10^{4}$ l.f.u./ml obtained when all three components were driven by the retroviral promoter (Table 1).

Despite the improvements made in retrovirus titer by the incorporation of the CMV promoter into the packaging components, we speculated that the level of vector genome RNA may be limiting the titers in this system. To address this possibility, we constructed CMV promoter-driven retroviral vectors. These retained the authentic RNA start site to ensure correct reverse
A.
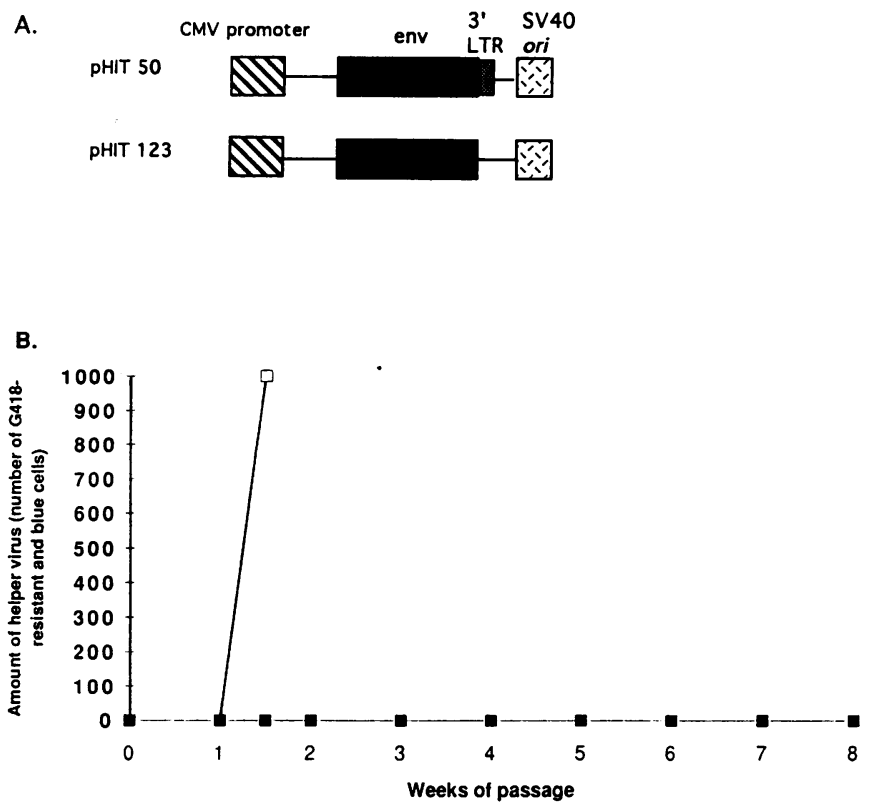

Figure 3. Effective elimination of helper virus formation. (A) Schematic representation of the ecotropic envelope constructs used in the helper virus assay. pHIT50 contains a small region of the $3^{\prime}$ LTR at the end of the envelope coding region. In pHIT123 the $3^{\prime}$ LTR sequences were removed by PCR. (B) Both helper virus assays were performed as described in the text and tested up to 8 weeks. Exchange of the envelope construct pHIT50 (open box) with pHIT123 (closed box) in the three-plasmid co-transfections eliminated helper virus production.

transcription of the vector genome following infection. An oligonucleotide was designed to precisely place the transcriptional start site for the CMV promoter at the +1 position found at the boundary of the U3/R sequences of the retroviral genome vectors (Fig. 1B). Both pLNSX and pLZSN were placed under the control of the CMV promoter and the resulting molecules were designated pHIT110 and pHIT111 respectively (Fig. 1). The correct start site for transcription of the retroviral genome was confirmed by primer extension analysis on total RNA extracted from 293T cells transfected with pHIT110 (Figs 1 and 2).

Co-transfection of all three CMV promoter-SV40 ori constructs, the pHIT plasmid series, generated the highest titers, which were in excess of $10^{6}$ infectious units/ml (Table 1). In keeping with our previous observations, pHIT110 generated slightly higher titers than pHIT111. At present, the cause of this effect is not clear, but it may be that the larger lacZ-neo transcript is packaged less efficiently. We are currently exploring the vector containing the smaller luc reporter gene as a means to overcome this disadvantage. The direct comparison of infectious titers from transfections with pHIT60, pHIT50 and pHIT111 as the vector genome $\left(1.4 \times 10^{6}\right.$ l.f.u. $\left./ \mathrm{ml}\right)$ versus $\mathrm{pRV} 114$ as the vector genome $\left(3 \times 10^{5}\right.$ l.f.u. $\left./ \mathrm{ml}\right)$ confirmed that expression of the vector genome by the CMV promoter increased virus titers (Table 1). We further quantitated virus titers obtained from various combinations of the LTR- and CMV-driven cassettes (Table 1). These studies confirmed that it was necessary to have all three components driven by the CMV promoter in order to achieve the highest titer retroviral stocks. The use of any one LTR plasmid reduced the titers accordingly. Similar relative titers were also obtained using COS- 1 cells, although levels were $\sim 10$-fold lower across all constructions (data not shown). 


\section{Elimination of helper virus by removal of $3^{\prime}$ overlapping regions}

We examined the supernatants from transfected 293T cells for the presence of contaminating replication-competent virus or helper virus. Undiluted supernatants of the highest titer retroviral stocks produced by co-transfection of 293T cells with pHIT60, pHIT50 and pHIT110 were used to infect NIH 3T3 cells and these cells were then passaged for 4 weeks to allow virus spread. At each passage, the culture supernatants were harvested, filtered and used to infect fresh NIH 3T3 cells. These cells were selected in G418 for up to 10 days to assay for the presence of replicationcompetent virus harboring the neo gene.

In addition, we used a sensitive marker rescue assay for the detection of helper virus by using an NIH 3T3 cell line stably transduced with pHIT111, which is similar to the previously reported BAG cell line (20). The integrated lacZ-containing genome in these cells can be rescued by transfection of the cells with the retroviral packaging components on plasmids pHIT60 and pHIT50 (data not shown). Medium from NIH 3T3 cells infected with supernatants from the transfected 293T cells was used to infect this cell line. Forty eight hours later, the supernatants from the cell line were filtered and used to infect fresh NIH 3T3 cells, which were then stained for $\beta$-galactosidase activity $24 \mathrm{~h}$ later. Any $\beta$-galactosidase-positive cells appearing at this stage are due to lac $Z$ genomes that had been rescued from the pHIT111 cell line by helper virus.

In a recent report by Pear et al. (21), replication-competent virus was found following three-plasmid co-transfection into 293T cells, although Landau and Littman (5) did not detect any replication-competent virus following transfection of COS-7 cells. We have detected helper virus at 10 days post-infection using both of the above assays. However, we have identified a small region of the $3^{\prime}$ LTR present in penv and pHIT50 that is homologous to the vector genomes and which may contribute to helper virus formation through three recombination events of our input plasmids. To eliminate the possibility of this occurring, we have removed the homologous overlap in pHIT50 by isolating only the p15e env sequences by $\mathrm{PCR}$ and replacing the corresponding sequences in pHIT50 containing the $3^{\prime}$ end of the env sequence and the $3^{\prime}$ LTR. The new CMV promoter-driven env expression plasmid lacking the homologous $3^{\prime}$ LTR sequences was designated pHIT123 (Fig. 3A). When the two helper virus assays were repeated using plasmids pHIT60, pHIT123 and pHIT110, G418-resistant colonies and rescue of the lacZ gene were undetectable even after 8 weeks of passage (Fig. 3B).

\section{Maximizing virus titers from transient transfections with NaB}

We have so far demonstrated that increases in virus titers were achieved by the use of SV40 ori-containing plasmids and by using the CMV promoter to drive both the structural and vector components of the system. The use of chloroquine, although recommended by Pear et al. (21), did not increase our titers on 293T cells (data not shown). We did, however, see a dramatic increase in virus titer after treatment of the transfected cells with $\mathrm{NaB}$ (Fig. 4). $\mathrm{NaB}$ has been shown to increase the percentage of cells expressing exogenous DNA (22), possibly through its effect on the cell cycle (23) or by inducing erythroid differentiation in hematopoietic cell lines (24-26). The effect of $\mathrm{NaB}$ on gene

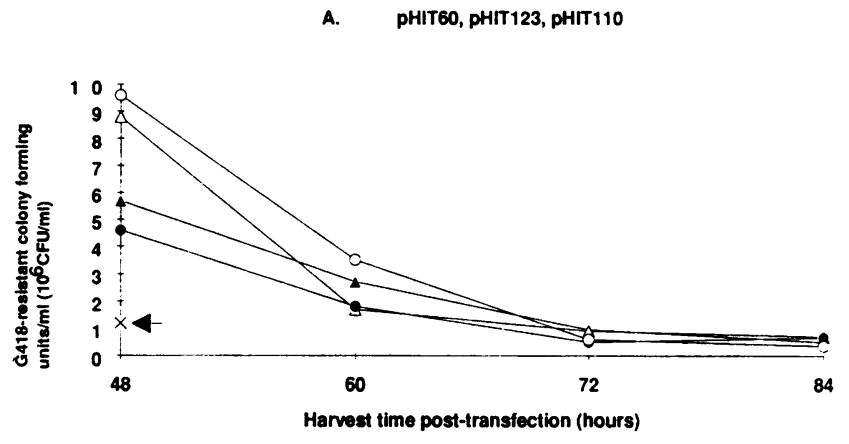

B. PHIT60, pHIT123, pHIT111

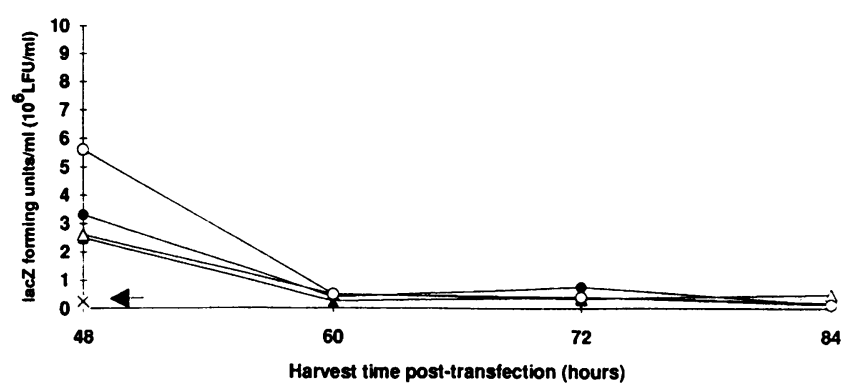

Figure 4. Effect of $\mathrm{NaB}$ on retrovirus titers. 293T cells were transfected and different concentrations of $\mathrm{NaB}$ were added directly to the medium following removal of the DNA-calcium phosphate co-precipitate. Viral supernatants from 293T cells transfected with (A) pHIT60, pHIT123 and pHIT110 or (B) pHIT60, pHIT123 and pHIT111 were harvested 48, 60, 72 and $84 \mathrm{~h}$ post-transfection and titered on NIH 3T3 cells. Final concentrations of $\mathrm{NaB}$ used were 2 (closed triangle), 5 (open triangle), 7 (closed circle) and $10 \mathrm{mM}$ (open circle). Arrows indicate titers obtained $48 \mathrm{~h}$ post-transfection without $\mathrm{NaB}$ treatment. Titers obtained were averages from two independent experiments and there was $\leq 4 \%$ variation between experiments.

expression has also been shown to be enhancer dependent (22) and a number of eucaryotic promoters are activated by $\mathrm{NaB}$, including the CMV promoter $(27,28)$. NaB inductions were therefore performed on 293T cells transfected with pHIT60, pHIT123 and pHIT110 or pHIT111 using $\mathrm{NaB}$ at final concentrations of $2,5,7$ and $10 \mathrm{mM}$. Figure 4 shows the average titers from two independent experiments determining the optimal time to harvest the supernatants and the $\mathrm{NaB}$ concentration which induced the highest titers. In every case titers peaked at $48 \mathrm{~h}$ post-transfection, but infectivity was greatly reduced at $60 \mathrm{~h}$ and titers were at least one log lower by $72 \mathrm{~h}$ post-transfection. A final concentration of $10 \mathrm{mM} \mathrm{NaB}$ enhanced viral titers by almost a log. Without $\mathrm{NaB}$ treatment, pHIT60, pHIT123, pHIT110 gave a titer of $1.2 \times 10^{6}$ c.f.u./ml and pHIT60, pHIT123, pHIT111 gave $2.5 \times 10^{5}$ l.f.u./ml as compared with $9.6 \times 10^{6}$ c.f.u./ml (Fig. $4 \mathrm{~A}$ ) and $5.6 \times 10^{6}$ l.f.u./ml (Fig. 4B) respectively after induction with $10 \mathrm{mM} \mathrm{NaB}$.

\section{Passive DNA transfer and retrofection}

The use of relatively high amounts of DNA in the 293T transfections raises the possibility that DNA may be transfered in the infection assay or that plasmid-encoded RNA may be transfered by non-specific retrofection. To test the first possibility, $10 \mu \mathrm{g}$ of pHIT111 or pSV2neo (29) were transfected into 293T 
cells and the supernatants were harvested and used to 'infect' NIH 3T3 cells as described above. The 'infected' NIH 3T3 cells were selected in $400 \mu \mathrm{g} / \mathrm{ml} \mathrm{G} 418$ for 10-15 days but no resistant colonies were observed (data not shown). Although free DNA may be carried over onto NIH 3T3 cells, it does not passively enter the cells and produce artificially high titers.

In retrofection, non-retroviral RNAs become encapsidated into the retroviral particles and become reverse transcribed upon infection (30,31). To test this possibility, pHIT60 and pHIT123 were transfected into 293T cells with either pHIT111 (a retroviral vector genome), pPE346 (a non-retroviral CMV-lacZ plasmid) (32) or pMW41 (a non-retroviral CMV-luc plasmid derived from pMW16 (33) into which the SV40 ori was inserted). As expected, co-transfection of pHIT60, pHIT123 and pHIT111 produced titers of $10^{5}$ l.f.u./ml without $\mathrm{NaB}$ treatment, whereas no lacZ-positive clones were seen with pPE346 and no luciferase activity was detected with pMW41. Therefore, the titers achieved using the HIT vectors are not due to the entry of free DNA into the cells by binding to virus particles nor to the non-specific encapsidation of RNAs.

\section{DISCUSSION}

We have reported here a new system for the production of high titer helper-free retroviral stocks by transient transfection. Under the optimized conditions described using the 293T cell line we achieved titers nearing $10^{7}$ infectious units $/ \mathrm{ml}$ at $48 \mathrm{~h}$ post-transfection. The viral stocks produced were helper virus-free, as no helper virus could be detected even after 8 weeks of passage. This system could be used for the transduction of cell lines or for the rapid screening of MLV gag-pol or env mutants.

In order to achieve maximum titers from retrovirus stocks by transient transfection, it is necessary to increase either or both viral gene expression and transfection efficiency of the producer cells. In an approach similar to that of Landau and Littman (5), we boosted gene expression in the first instance by the combined use of SV40 ori sequences in all the retroviral components and host cells expressing the SV40 large T antigen. 293T cells were used as they have previously been reported to be highly transfectable (21). We further improved gene expression of the packaging components (gag-pol and env) in the system by directing their expression from the $\mathrm{CMV}$ promoter. We have also demonstrated that driving transcription of the vector genome from the CMV promoter further increased infectious titers. The highest titers were obtained when all three components were on CMV-driven plasmids and the use of any one component driven by the LTR limited the infectious titer. This appeared especially true for the env component in the system, as expression of this gene from the LTR promoter had the most severe effect on the infectious titer of the virus stock. Finally, a study was undertaken to maximize the yields of virus by inducing expression of the CMV promoter-driven plasmids with $\mathrm{NaB}$.

When the virus stocks were screened for helper virus, helper virus was initially detected in virus stocks generated using the CMV promoter-driven env expression vector pHIT50. This plasmid contains a small region of the $3^{\prime}$ LTR that creates a homologous overlap with the vector genomes. Removal of this homologous overlap effectively eliminated helper virus formation.

This is the first reported method for obtaining $\sim 107$ helper-free viral stocks without the need to establish and maintain packaging cell lines or stable producer lines, thereby allowing rapid characterization of gag-pol and env mutants and producing high titer retroviral vectors for transduction by a convenient, rapid and reproducible method. During the course of this work, a similar system was reported (34), although titers appeared to be somewhat lower. We have also developed a CMV promoterdriven amphotropic envelope system that may have applications in human gene therapy.

\section{ACKNOWLEDGEMENTS}

We thank David Baltimore for providing the 293T cells, Dusty Miller for the pLNSX, pLZSN and pLNCX plasmids and Martin Braddock and Nigel Clark for helpful discussions. This work was supported by a grant from British Biotechnology Ltd.

\section{REFERENCES}

1 Miller,A.D. (1990) Hum. Gene Ther., 1, 5-14.

2 Sorge,J., Wright,D., Erdman,V.D. and Cutting,A.E. (1984) Mol. Cell. Biol., 4, 1730-1737.

3 Miller,A.D. and Buttimore,C. (1986) Mol. Cell. Biol., 6, 2895-2902.

4 Bender,M.A., Palmer,T.D., Gelinas,R.E. and Miller,A.D. (1987) J. Virol., 61, 1639-1646.

5 Landau,N.R. and Littman,D.R. (1992) J. Virol., 66, 5110-5113.

6 Boshart,M., Wever,F., Jahn,G., Dorsch-Häsler,K., Fleckenstein,B. and Schaffner,W. (1985) Cell, 41, 521-530.

7 Foecking,M.K. and Hofstetter,H. (1986) Gene, 45, 101-105.

8 DuBridge,R.B., Tang,P., Hsia,H.C., Leong,P.-M., Miller,J.H. and Calos,M.P. (1987) Mol. Cell. Biol., 7, 379-387.

9 Markowitz,D., Goff,S. and Bank,A. (1988) J. Virol., 62, 1120-1124.

10 Miller,A.D. and Rosman,G.J. (1989) Biotechniques, 7, 980-990.

11 Adam,M.A., Ramesh,N., Miller,A.D. and Osborne,W.R.A. (1991) J. Virol., 65, 4985-4990.

12 Boulter,C.A. and Wagner,E.F. (1987) Nucleic Acids Res, 15, 7194.

13 Chapman,B.S., Thayer,R.M., Vincent,K.A. and Haigwood,N.L. (1991) Nucleic Acids Res., 19, 3979-3986.

14 Shinnick,T.M., Lerner,R.A. and Sutcliffe,J.G. (1981) Nature, 293, 543-548.

15 Ogden,J.E., Stanway,C., Kim,S., Mellor,J., Kingsman,A.J. and Kingsman,S.M. (1986) Mol. Cell. Biol., 6, 4335-4343.

16 Adams,S.E., Johnson,I.D., Braddock,M., Kingsman,A.J., Kingsman,S.M. and Edwards,R.M. (1988) Nucleic Acids Res., 16, 4287-4298.

17 Graham,F.L. and Van der Eb,A.J. (1973) Virology, 52, 456-467.

18 Sanes,J.R., Rubenstein,J.L.R. and Nicolas,J. (1986) EMBO J., 5, 3133-3142.

19 Braddock,M., Thorburn,A.M., Chambers,A., Elliot,G.D., Anderson,G.J., Kingsman,A.J. and Kingsman,S.M. (1990) Cell, 62, 1123-1133.

20 Price,J., Turner,D. and Cepko,C. (1987) Proc. Natl. Acad. Sci. USA, 84, 156-160.

21 Pear,W.S., Nolan,G.P., Scott,M.L. and Baltimore,D. (1993) Proc. Natl. Acad. Sci. USA, 90, 8392-8396.

22 Gorman,C.M. and Howard,B.H. (1983) Nucleic Acids Res., 11, 7631-7648.

23 D’Anna,J.A., Tobey,R.A. and Gurley,L.R. (1980) Biochemistry, 19, 2656-2671.

24 Hock,R.A., Miller,A.D. and Osborne,W.R.A. (1989) Blood, 74, 876-881.

25 Leder,A. and Leder,P. (1975) Cell, 5, 319-322.

26 Reeves,R. and Cserjesi,P. (1979) J. Biol. Chem., 254, 4283-4290.

27 Radsak,K., Fuhrmann,R., Franke,R.P., Schneider,D., Kollert,A., Brücher,K.H. and Drenckhahn,D. (1989) Arch. Virol., 107, 151-158.

28 Tanaka,J., Sadanari,H., Sato,H. and Fukuda,S. (1991) Virology, 185, 271-280.

29 Southern,P. and Berg,P. (1982) J. Mol. Appl. Genet., 1, 327.

30 Linial,M. (1987) Cell, 49, 93-102.

31 Levine,K.L., Steiner,B., Johnson,K., Aronoff,R., Quinton,T.J. and Linial,M.L. (1990) Mol. Cell. Biol., 10, 1891-1900.

32 Blanchard,A.D., Powell,R., Braddock,M., Kingsman,A.J. and Kingsman,S.M. (1992) J. Virol., 66, 6769-6772.

33 White,M.R.H., Masuko,M., Amet,L., Elliot,G., Braddock,M., Kingsman,A.J. and Kingsman,S.M. (1995) J. Cell Sci., in press.

34 Finer,M.H., Dull,T.J., Qin,L., Farson,D. and Roberts,M.R. (1994) Blood, 83, 43-50. 